\title{
Basal body and flagellum mutants reveal a rotational constraint of the central pair microtubules in the axonemes of trypanosomes
}

\author{
Catarina Gadelha ${ }^{1, \star}$, Bill Wickstead ${ }^{1, \star}$, Paul G. McKean ${ }^{2}$ and Keith Gull, ${ }^{1, \neq}$ \\ ${ }^{1}$ Sir William Dunn School of Pathology, University of Oxford, South Parks Road, Oxford, OX1 3RE, UK \\ ${ }^{2}$ Biomedical Sciences Unit, Department of Biological Sciences, Lancaster University, Lancaster, LA1 4YQ, UK \\ ${ }^{*}$ These authors contributed equally to this work \\ ${ }^{\ddagger}$ Author for correspondence (e-mail: keith.gull@ path.ox.ac.uk) \\ Accepted 2 March 2006 \\ Journal of Cell Science 119, 2405-2413 Published by The Company of Biologists 2006 \\ doi:10.1242/jcs.02969
}

\section{Summary}

Productive beating of eukaryotic flagella and cilia requires a strict regulation of axonemal dynein activation. Fundamental to any description of axonemal beating is an understanding of the significance of the central pair microtubules and the degree to which central pair rotation has a role. However, for the majority of organisms, it is unclear whether the central pair actually rotates. Using an extra-axonemal structure as a fixed reference, we analysed the orientation of the central pair in African trypanosomes and other kinetoplastid protozoa. A geometric correction allowed the superposition of data from many cross-sections, demonstrating that the axis of the central pair is invariant and that there is no central pair rotation in these organisms. Analysis of mutants depleted in particular flagellar and basal body proteins $[\gamma$-tubulin, $\delta$-tubulin, Parkin co-regulated gene product (PACRG) or the paraflagellar rod protein PFR2] allowed a dissection of the mechanisms for central pair constraint. This demonstrated that orientation is independent of flagellum attachment and beating, but is influenced by constraints along its length and is entirely dependent on correct positioning at the basal plate.

Key words: Axoneme, Flagellum, Central pair, Paraflagellar rod, Delta-tubulin, Basal body

\section{Introduction}

In eukaryotes, the motility of flagella and cilia results from a microtubule-based assembly known as the axoneme. Although a variety of microtubule arrangements exist in motile flagella, the canonical axoneme - the structure most widespread and almost certainly ancestral to all others (Cavalier-Smith, 2002; Cavalier-Smith and Chao, 2003) - consists of nine microtubule doublets symmetrically arranged in a circle around a central pair (CP) of singlet microtubules (the so-called $9+2$ arrangement). This structure is anchored at the proximal end by a basal body containing triplet microtubules in a $9+0$ arrangement. Dynein arms are attached to the A-tubule of each doublet such that their motor head domains are in close proximity to the B-tubule of the neighbouring doublet (Gibbons and Gibbons, 1973; Gibbons and Rowe, 1965). Activation of the dynein motors causes a sliding force between adjacent doublets (Sale and Satir, 1977). Because the microtubules are constrained at the basal body and along their length, this force is translated into an axonemal bend. This is the fundamental force of axonemal motion.

From even such a basic description of axonemal action, one can recognise the need for three kinds of regulation of dynein activation to create a productive axonemal beat. (1) Asymmetric activation: given the symmetry of the outer doublets, the dynein arms on one side must be active while those on the other are quiescent to generate a productive bend. (2) Co-operative activation: a bend of any significant length requires the simultaneous (in)activation of many dynein molecules along that stretch of axoneme. (3) Dynamic activation: the propagation of a bend along the axoneme requires that dyneins in a particular axonemal region be coherently switched between active and inactive states.

One model for the regulation of dynein activation is based on evidence that indicates a core role for the $\mathrm{CP}$ microtubules. In this model, signal from an asymmetric $\mathrm{CP}$ apparatus (consisting of the two singlet microtubules $\mathrm{C} 1$ and $\mathrm{C} 2$, and associated structures such as the $\mathrm{CP}$ bridge and $\mathrm{CP}$ projections) is transduced through the radial spokes, to a doublet-associated regulatory complex, which modifies dynein activity through the action of dynein-associated protein kinases and phosphatases (Porter and Sale, 2000; Smith and Yang, 2004). In support of this model, asymmetric contacts between radial spokes and CP projections have been seen in molluscan gill cilia (Warner and Satir, 1974), suggesting that radial spokes might detach and reattach to the $\mathrm{CP}$ projections during microtubule sliding. In Chlamydomonas, $\mathrm{CP}$ mutants are paralysed yet retain the ability to undergo microtubule sliding; their defect is therefore not in the force-generating mechanism but in a control system that regulates force generation (reviewed by Smith and Lefebvre, 1997).

The CP signal transduction model is particularly significant in the context of $\mathrm{CP}$ rotation. It has been postulated that rotation of an asymmetric $\mathrm{CP}$ could, through interactions between one set of $\mathrm{CP}$ projections and radial spokes, regulate 
the activation of dyneins on successive doublets around the axoneme. By contrast, associations made by the opposing $\mathrm{CP}$ microtubule would ensure that dyneins on the opposite side of the axoneme were inactive. The $\mathrm{CP}$ would then act as a distributor of regulatory signals around the axoneme.

$\mathrm{CP}$ rotation during beating of flagella and cilia has been demonstrated by thin-section electron microscopy in Chlamydomonas and Paramecium. Longitudinal sectioning through Chlamydomonas flagella that were fixed during active swimming revealed the orientation of the $\mathrm{CP}$ microtubules as being parallel to the bend plane within the principal bend, then gradually twisting through $180^{\circ}$ until once again being parallel to the bend plane within the reverse bend (Mitchell, 2003). In fact, the CP assumes a natural left-handed helix when separated from the rest of the axoneme by detergent (Goodenough and Heuser, 1985) and protease treatments (Hosokawa and MikiNoumura, 1987). It thus seems reasonable to accept that the $\mathrm{CP}$ is also helical inside the native axoneme, and that rotation of the helix through successive waves allows the CP to reorient such that one of the two CP microtubules is always at the outside of the bend. However, this $\mathrm{CP}$ rotation is independent of both dynein activation (as it also occurs in curved, quiescent flagella) and also spoke head association (as it occurs during bend propagation in spoke head mutants) (Mitchell and Nakatsugawa, 2004). Hence, CP orientation is a passive response to bend formation in Chlamydomonas.

$\mathrm{CP}$ orientation in Paramecium cilia is also parallel to the plane of bending, and it rotates $360^{\circ}$ during each beat cycle in forward and backward swimming (Omoto and Kung, 1979; Omoto and Kung, 1980). Serial thin sections also revealed the CP lefthanded twist along the Paramecium axoneme. In contrast to Chlamydomonas quiescent flagella, paralysed paramecia do not show CP rotation (Omoto and Kung, 1980). Therefore, rather than being a passive process, rotation of the $\mathrm{CP}$ in Paramecium might be an active one, initiated or constantly driven by a mechanism other than bending, with the orientation of the $\mathrm{CP}$ determining the pattern of active sliding around the axoneme (Omoto and Kung, 1980). It is worth noting that both Chlamydomonas and Paramecium rotate their CP tubules clockwise when viewed from the base of the flagellum.

However, CP rotation is not ubiquitous in axonemal beating. In both ctenophore comb plate cilia and echinoderm sperm flagella, the CP apparatus has a fixed orientation relative to the position of active sliding (Sale, 1986; Tamm and Tamm, 1981). For most organisms, it is not yet clear if the CP rotates. This is largely a result of the difficulties in ultra-structural analyses of imposing an absolute frame of reference onto the ninefold symmetric doublets of the axoneme, making it problematic to perceive a change in the axis of the $\mathrm{CP}$ relative to the doublets. This situation is considerably eased in kinetoplastid protozoa owing to the presence in the flagellum of a characteristic extraaxonemal structure - the paraflagellar rod (PFR). Here, we use this structure as an external reference to address the orientation of the CP in the flagella of several kinetoplastid species. We show that there is no $\mathrm{CP}$ rotation and use a simple geometric correction technique to produce an accurate estimate of the angle of the CP axis in Trypanosoma brucei from a large number of axonemal cross-sections. We also present a first examination of the causes of this constraint using RNA interference (RNAi) to deplete particular flagellar and basal body proteins specifically.

\section{Results}

\section{Fixed CP orientation in T. brucei}

In Kinetoplastida, the PFR runs alongside the axoneme for most of its length (Fuge, 1969; Gull, 1999). Importantly, the PFR is fixed relative to the axoneme, being permanently connected to a set of four outer doublets (Gull, 1999). The PFR therefore acts as a reference, allowing unambiguous identification of $\mathrm{CP}$ orientation to an external axis within the flagellum.

To determine the $\mathrm{CP}$ orientation in the kinetoplastid flagella, we analysed 40 randomly selected thin-section transmission electron micrographs showing cross-sections of axonemes from motile procyclic-form $T$. brucei. Most micrographs showing near 'transverse' sections through an axoneme cannot be directly superimposed because there is inevitably a slight (and often a substantial) discrepancy between the angle of the section and the true transverse plane. The result is an apparent deformation of the axoneme (Fig. 1A). To compensate for axonemal cross-sections not being true perpendicular sections, we used a mathematical transformation that moves the data into the transverse plane (Fig. 1B; detailed in Materials and Methods). Fig. 1C shows how this transformation can be used
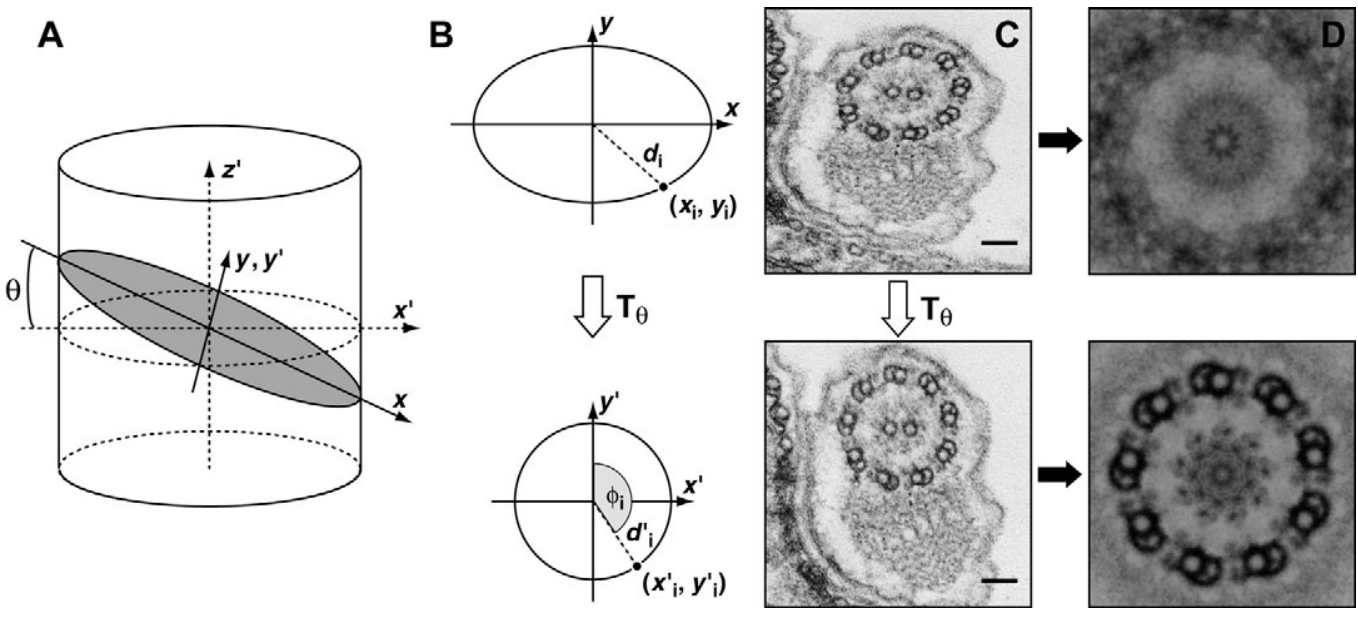

Fig. 1. (A) An elliptical section is formed if a perfect cylinder with longitudinal axis $z^{\prime}$, is cut at an angle $\theta$ to the $x^{\prime} y^{\prime}$ plane. (B) The ellipse can be transposed onto the $x^{\prime} y^{\prime}$ plane by a transformation, $\mathrm{T}_{\theta}$, to generate a true transverse section. (C) A thin-section transmission electron micrograph showing a crosssection through a $T$. brucei flagellum. The section angle was corrected by the transformation $\mathrm{T}_{\theta}$ to generate a transverse section. (D) Ninefold rotation of the corrected axoneme shown in C. Bars, $50 \mathrm{~nm}$. 
to correct an elliptical axonemal cross-section to a circular one representative of a true transverse section. The circularity of the transformed dataset can be demonstrated by ninefold rotation of the corrected image, showing superposition of the doublets of the axoneme (Fig. 1D).

We should note that this ellipse-correction procedure would also erroneously 'correct' any symmetric deformation of the axoneme in a true transverse section caused by bending forces. However, since the transformation is applied only once, it cannot correct for both a symmetric deformation and a glancing section. Since there is very little eccentricity in the transformed dataset (Fig. 1C), whereas the original crosssections were often highly elliptical (eccentricities up to 0.8 ; i.e. the ellipse long axis is nearly twice that of the short axis), it is unlikely that deformation of the axoneme as a result of beating is large in these micrographs when compared with eccentricity as a result of sectional plane. Bending waves can be clearly seen in the fixed flagella when interrogated by light microscopy (data not shown).

Following transformation of the data from the micrographs, the positions of all of the microtubules are extremely constant from axoneme to axoneme (Fig. 2A) and fall on the points predicted on the basis of perfect ninefold symmetry. Using the PFR as an external reference to define the doublet number (by being attached to doublets 4-7), the axis of the CP also has a very constant orientation at $92.7 \pm 0.5^{\circ}$ (mean \pm s.e.m.) to the axis defined by the centre of the axoneme and doublet 1 (Fig. 2A,B). Thus, the orientation of the $\mathrm{CP}$ does not vary with respect to the nine outer doublets. Since this dataset comprises cross-sections through axonemes of many different cells and at different positions along the cell, this provides strong evidence for a fixed, specific orientation for the $\mathrm{CP}$ in $T$. brucei, precluding $\mathrm{CP}$ rotation in this species. We therefore propose that the mechanism by which the $\mathrm{CP}$ might rotate with respect to the outer nine doublet microtubules in some species does not operate in trypanosomes.
Fixed CP orientation in other Trypanosomatidae

In T. brucei, the single flagellum is physically attached to the cell body along most of its length through the flagellum attachment zone, a precise system of membrane connections, filaments and specialised microtubules (Gull, 1999). However, the trypanosomatids in general can display attached, partially attached and free flagella, all of which possess the external reference of the PFR. We next asked whether the fixed orientation of the CP in the axonemes of T. bruce $i$ was specific to this species, or indeed life-cycle stage, or if it was common to other species and cell types.

The same geometric correction demonstrated in Fig. 1 for procyclic-form $T$. brucei axonemes was performed on bloodstream-form $T$. brucei cells. These two life-cycle stages move very differently from one another, yet the $\mathrm{CP}$ of each was found to be fixed in the same orientation (Fig. 3A,B). The same is also true for axonemes of epimastigote-form T. cruzi semiattached flagella (Fig. 3C), as well as Leishmania major, Herpetomonas megaseliae and Crithidia fasciculata free flagella (Fig. 3D-F). Once more, the position of the CP was invariant and had the same orientation as that seen in T. brucei.

The most recent common ancestor of the above kinetoplastid species existed $\sim 250$ million years ago (Douzery et al., 2004) and, in the light of these data, most probably had a $\mathrm{CP}$ in a fixed orientation. Moreover, in spite of many differences in cell morphology, it appears that all of the extant progeny from this common ancestor have maintained the machinery that constrains the $\mathrm{CP}$ of microtubules.

\section{Release of CP constraint in mutants}

We next asked what factors were controlling the fixed $\mathrm{CP}$ orientation seen in trypanosomes. We used double-stranded RNAi to deplete particular flagellar and basal body proteins to gain insight into the mechanism determining $\mathrm{CP}$ orientation in T. brucei. The first of these was $\delta$-tubulin - a protein associated with basal bodies. In Chlamydomonas, disruption of the gene
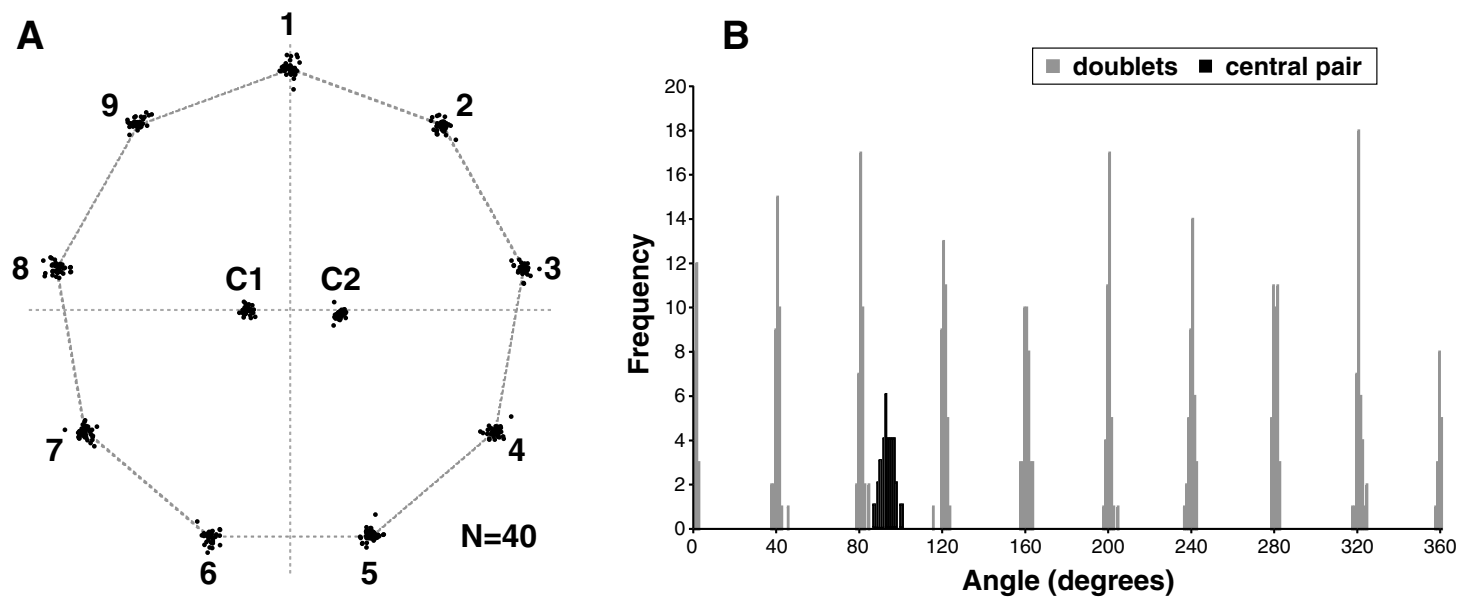

Fig. 2. (A) The positions of microtubules in transverse sections of axonemes from $T$. brucei. Points show the positions of the centres of either the $\mathrm{CP}$ microtubules or the A-tubules of the outer doublets. Dotted lines show the $x^{\prime}$ and $y^{\prime}$ axes of the axoneme (see Materials and Methods) and also a regular normal nonagon. The number, N, indicates the number of independent micrographs combined in the diagram. (B) Radial distribution of the doublets and the CP microtubules in transverse sections of axonemes from the wild-type trypanosomes. Since the CP are indistinguishable on the electron micrographs, their distribution can be anywhere in the range $0-180^{\circ}$, whereas the distribution of the doublets is $0-360^{\circ}$. 


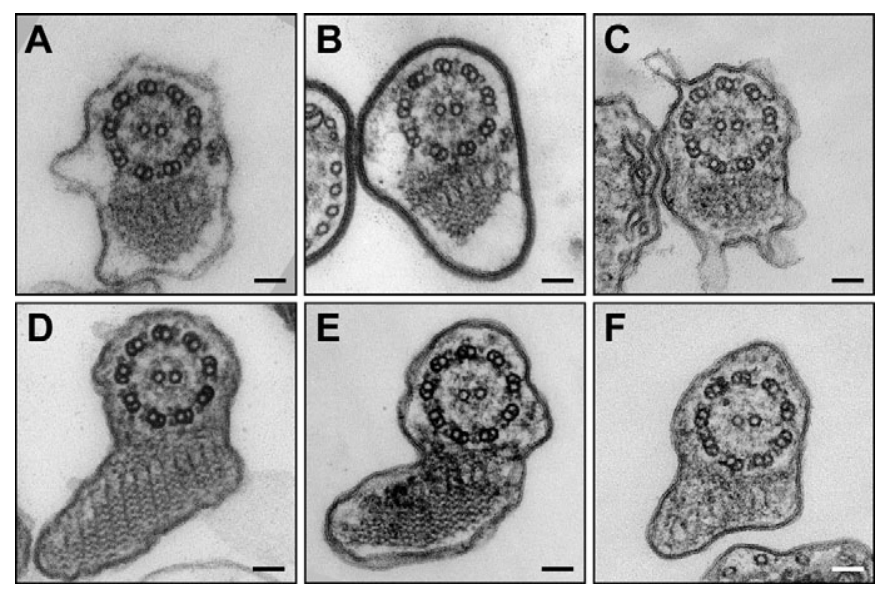

Fig. 3. The orientation of the CP microtubules is fixed, and at the same position, in Kinetoplastida species. Representative crosssection micrographs through the flagellum of (A) procyclic-form $T$. brucei, (B) bloodstream-form T. brucei, (C) epimastigote-form $T$. cruzi, (D) promastigote-form L. major, (E) promastigote-form $H$. megaseliae, and (F) choanomastigote-form $C$. fasciculata. These micrographs had the section angle corrected by the transformation $\mathrm{T}_{\theta}$ to become true transverse section, and they show the $\mathrm{CP}$

microtubules always aligned with respect to the doublets 3 and 8 . This orientation does not vary in either attached or partially attached flagella (trypanosomes), or free flagella (Leishmania, Herpetomonas, Crithidia). Bars, $50 \mathrm{~nm}$.

encoding $\delta$-tubulin results in loss of the C-tubule of the basal body microtubules (Dutcher and Trabuco, 1998; Fromherz et al., 2003). In Paramecium, silencing of the gene encoding $\delta$ tubulin similarly results in loss of the C-tubule, but additionally loss of B- and even A-tubules was observed (Garreau de Loubresse et al., 2001). Since the radial symmetry of the axoneme is born from the ninefold symmetry of the basal body, with the nine doublets of the axoneme extending from the A and $\mathrm{B}$ tubules of the triplet basal body, there is a prediction that disruption of the basal body configuration will be transferred along the axoneme.

We generated a $T$. brucei cell line for the inducible knockdown of $\delta$-tubulin by RNAi. Prior to induction of $\delta$ tubulin RNAi, trypanosomes were morphologically normal, and showed normal growth and motility. Induction of $\delta$-tubulin RNAi resulted in a reduction in growth rate after 48 hours (Fig. 4A). Several characteristic phenotypes were noted in trypanosome populations following induction of $\delta$-tubulin RNAi that varied in severity as a function of time postinduction (see below). The earliest phenotypic effect we observed, at $>24$ hours post-induction, was the loss of flagellar motility in trypanosomes that otherwise appeared morphologically normal (Fig. 4B). To confirm the efficacy of $\delta$-tubulin RNAi, we raised a monoclonal antibody, LAZ1, against $T$. brucei $\delta$-tubulin (see Materials and Methods) and used it to monitor the levels of $\delta$-tubulin protein post-induction. Immunoblotting confirmed that, following induction of RNAi, levels of $\delta$-tubulin were progressively reduced such that, by 48 hours post-induction, $\delta$-tubulin protein is barely detectable (Fig. 4C). By contrast, levels of $\beta$-tubulin - which acts as both loading and specificity control in these RNAi experiments - were unaffected during the time course of the experiment (Fig. 4C).

Induction of RNAi in these cells did not initially block flagellar construction but resulted in the production of aberrant basal bodies subtending immotile flagella. Basal body structure was reduced to combinations of triplet, doublet and singlet microtubules (Fig. 4D), showing that the trypanosome basal body is fundamentally dependent upon $\delta$-tubulin function. Distal from the basal bodies, ultra-structural analysis of flagella revealed disruption of the $9+2$ arrangement by loss of outer doublets (Fig. 4F,G). At later time-points post-induction ( $>96$ hours), more-severe defects were observed (with several doublets missing from the axoneme); however, at 48 hours post-induction, the majority of disrupted axonemes had lost only one doublet and in some instances only the B-tubule of a single doublet was absent. Interestingly, there was a significant preference for the disappearance of outer doublets 8 and $9\left(\chi^{2}\right.$ test, $P<0.001)$. Despite this loss of outer doublets, no loss of the $\mathrm{CP}$ occurred, indicating that $\mathrm{CP}$ nucleation is unimpaired in these cells.

Some axonemes in the induced $\delta$-tubulin RNAi cell line display the canonical $9+2$ arrangement. These most probably represent axonemes constructed before $\delta$-tubulin levels had dropped below a crucial level and, accordingly, their abundance decreases with increasing time post-induction (data not shown). In these axonemes, as for the non-induced cells, the CP was orientated as seen for wild-type T. brucei (Fig. 4E). However, loss of one outer doublet microtubule, or even just the B-tubule of a doublet, results in a total loss of correct CP orientation with the $\mathrm{CP}$ now being orientated apparently randomly around the central axis of the axoneme (Fig. 4F,G and Fig. 5D).

Another member of the tubulin family is $\gamma$-tubulin, also seen at the basal body and centriole region in a wide range of eukaryotes (Nigg, 2004). More precisely, $\gamma$-tubulin localises to the basal plate in Chlamydomonas, where it plays a role in CP nucleation (Silflow et al., 1999). We have previously reported the effects of $\gamma$-tubulin depletion in T. brucei-induction of $\gamma$ tubulin RNAi produces immotile flagella that lack the CP (McKean et al., 2003). However, early in $\gamma$-tubulin RNAi induction, there are a small number of cells ( 9\%) in which only one of the two CP microtubules is lost. We analysed these axonemes with respect to orientation of the remaining microtubule and found that, in these $9+1$ axonemes, the single central microtubule had a random distribution (Fig. 5A). Hence, although $\gamma$-tubulin RNAi does not affect the integrity of the nine outer doublets, the radial symmetry of the 9+1 arrangement is not sufficient for constraining the CP position. Interestingly, the remaining, mis-orientated central microtubule is found at the same distance from the centre of the axoneme, as it would be had its partner still been present, and does not collapse inwards. This argues against a model in which the CP push against the radial spokes to create a force that is transduced out to the doublets.

Unlike the basal body proteins $\gamma$ - and $\delta$-tubulin, the T. bruce $i$ Parkin co-regulated gene products TbPACRG A and TbPACRG $\mathrm{B}$ are related proteins that appear to have a role in doublet morphogenesis and locate along the length of the flagellum (Dawe et al., 2005). Depletion of both homologues simultaneously (although not separately) by RNAi in T. brucei causes flagellar paralysis and leads to premature termination of 

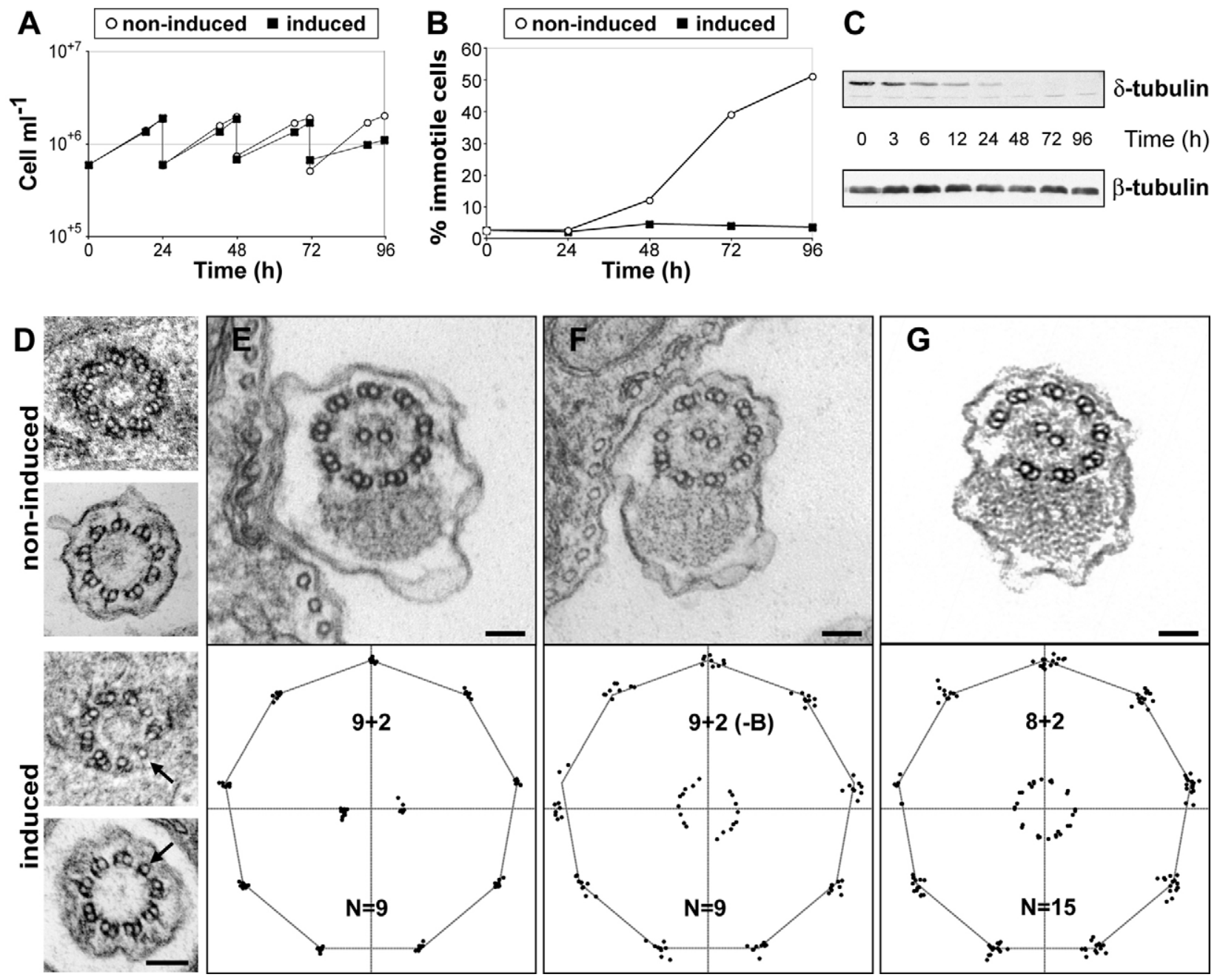

Fig. 4. $\delta$-tubulin ablation in T. brucei results in growth rate reduction, cell paralysis, aberrant basal bodies and disrupted CP orientation. Growth (A) and motility (B) of $\delta$-tubulin RNAi mutant cells with (closed squares) and without (open circles) tetracycline induction. (C) Western blot analysis of $\delta$-tubulin depletion. Total cell lysates prepared at various time-points after $\delta$-tubulin RNAi induction were separated by SDS-PAGE and analysed with mAb LAZ1 (raised against $\delta$-tubulin) or mAb KMX (reacts with $\beta$-tubulin). Each lane contains the protein from $2 \times 10^{6}$ cells. (D) The microtubule number in the basal bodies of the T. brucei $\delta$-tubulin RNAi cell line. Non-induced basal bodies possess the canonical nine-triplet arrangement at the proximal region, and the normal nine-doublet arrangement at the transition zone. Induced basal bodies were reduced to a mixture of triplets, doublets and singlets. Arrows indicate singlet microtubules. Bar, $100 \mathrm{~nm}$. (E-G) The position of microtubules in transverse sections of induced T. brucei $\delta$-tubulin RNAi axonemes. Representative individual micrographs illustrate (E) a 9+2 axoneme, (F) an axoneme that lost the B-tubule of doublet number $8(9+2-\mathrm{B})$, and $(\mathrm{G})$ an axoneme that lost doublet number $7(8+2)$, and the corresponding position of microtubules within the respective nonagons. The number, $\mathrm{N}$, indicates the number of independent micrographs combined in the diagram. Bars, $50 \mathrm{~nm}$.

outer doublets - axoneme formation is initially normal, but the number of missing doublets increases towards the distal tip of the flagellum. The resulting disrupted axonemes look superficially like $\delta$-tubulin mutants but the defects are more severe and, importantly, do not originate in the basal body. Fig. $5 \mathrm{~B}$ shows the orientation of the $\mathrm{CP}$ in axonemes with variable numbers of outer doublets $(\mathrm{X}+2)$ resulting from PACRG ablation. Disruption of the axoneme again produced a perturbation of $\mathrm{CP}$ orientation. However, despite producing a more severe axonemal disruption than that of $\delta$-tubulin RNAi, $55 \%$ of the $\mathrm{X}+2$ axonemes analysed maintained the $\mathrm{CP}$ in a fixed, wild-type orientation. These data indicate a differential effect of axonemal disruptions originating at or near the basal plate compared with those that occur along the length of the flagellum.

Since the above mutants all result in flagellar motility defects, it was possible that $\mathrm{CP}$ orientation might be entrained by the intrinsic beating of the flagellum. We addressed this issue by analysing micrographs from a previously described RNAi against PFR2 in T. brucei (Bastin et al., 2000; Bastin et al., 1998). In these cells, interference in the building of a normal PFR structure leads to cell paralysis apparently without disruption of the axoneme itself. However, sufficient residual PFR structure remains for it still to be used as an external 

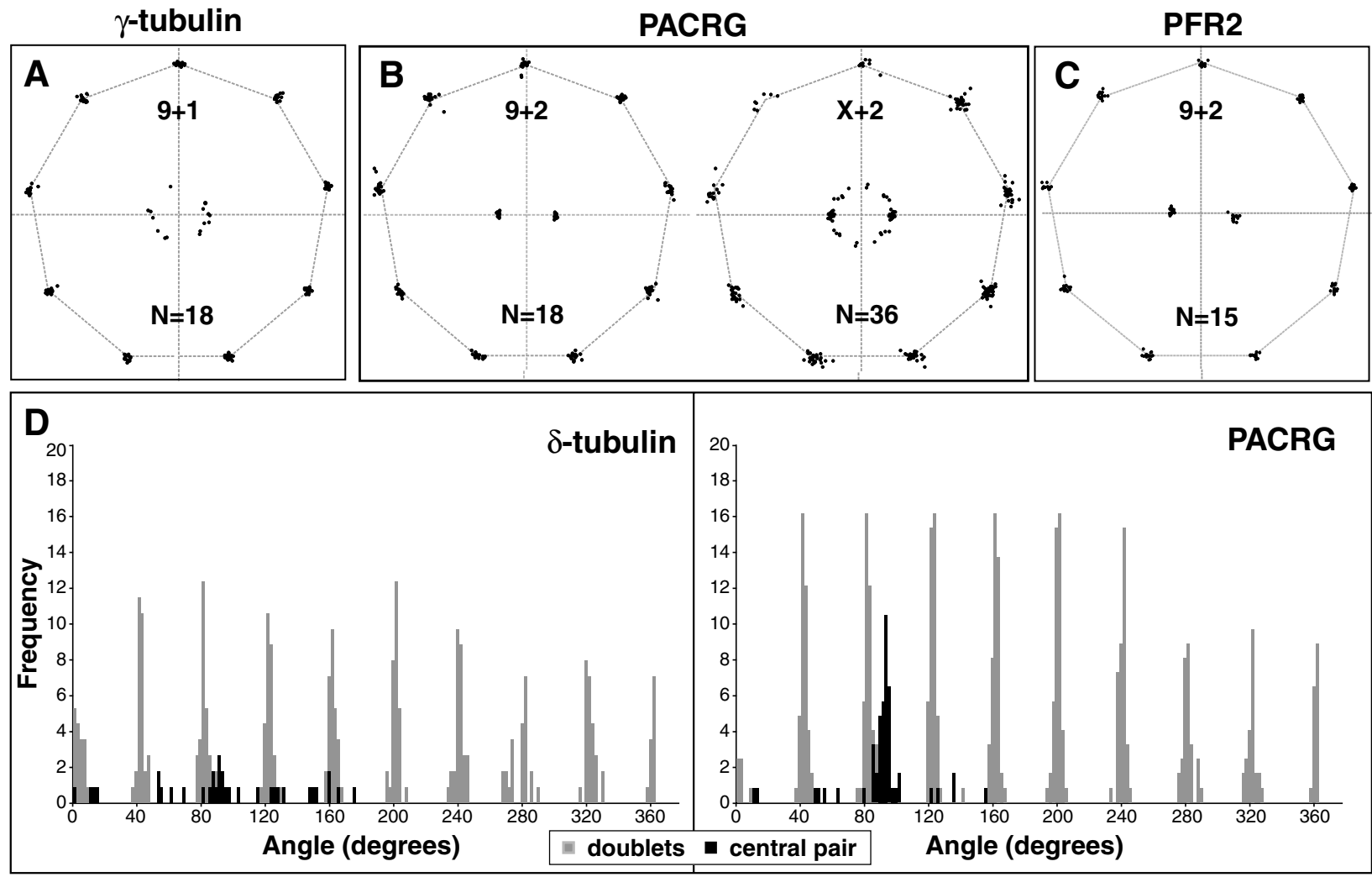

Fig. 5. CP orientation in $\gamma$-tubulin, PACRG and PFR2 RNAi mutant cell lines. (A) The remaining CP microtubule in 9+1 axonemes resulting from $\gamma$-tubulin ablation has a random orientation. (B) Knockdown of PACRG severely disrupts outer doublet microtubules, but only partially removes $\mathrm{CP}$ constraint in $\mathrm{X}+2$ axonemes. (C) Immotility as a result of PFR2 ablation does not affect CP orientation. (D) Radial distribution of the doublets and the $\mathrm{CP}$ microtubules in transverse sections of axonemes from the induced $\delta$-tubulin and PACRG RNAi trypanosomes.

reference to assign $\mathrm{CP}$ orientation, and examination of axonemal cross-sections reveals that the $\mathrm{CP}$ remains fixed (Fig. $5 \mathrm{C})$.

\section{Discussion}

We have used the singularity of the PFR structure to investigate orientation of the $\mathrm{CP}$ in a eukaryotic flagellum. The data presented here show a fixed $\mathrm{CP}$ orientation throughout the length of the axonemes in several trypanosomatid species and do not, therefore, support the hypothesis of $\mathrm{CP}$ rotation in Kinetoplastida. However, one must always be mindful in such ultra-structural studies of the possibility of fixation artefacts. Three lines of reasoning lead us to conclude that the fixed angle of the $\mathrm{CP}$ in our analysis reflects the true orientation of the $\mathrm{CP}$ during flagellar beating: (1) In this report, cells were fixed in aldehyde-based solutions believed to preserve the metachrony of the cilia on the Paramecium surface, as well as to preserve waveforms during forward and reverse swimming of Chlamydomonas. We reason that these reagents used to establish CP orientation in the latter two organisms were appropriate fixatives for our analysis in trypanosomes. Our conclusion of a fixed CP orientation is also compatible with data presented in previous studies on trypanosomes that involved other fixation techniques, such as high-pressure freezing and freeze substitution (Wiese et al., 2003), as well as detergent extraction followed by chemical fixation (Maga et al., 1999). (2) If the CP were falling back to a low-energy configuration within the axoneme, it might be expected that the precise orientation would be influenced by the degree of local strain as a result of bending. However, the axis of the CP is exceptionally well defined in our analysis despite being based on a number of samples from random points along flagella presumably possessing different torsional strains. (3) The fixed orientation of the CP in T. brucei can be disrupted by RNAi against specific gene products. This suggests that, were the $\mathrm{CP}$ of wild-type cells to rotate, our analysis would detect the rotation. However, although our and similar chemical-fixation techniques are fast enough to preserve both $\mathrm{CP}$ orientation and a normal-looking bending wave, it is noteworthy that they are probably not sufficiently rapid to capture an instantaneous 'snap-shot' of the transitory positions of every axonemal structure. For example, we cannot detect in our corrected axonemes any variation in the doublet positions caused by activated dyneins pulling doublets closer together - a central tenet to the geometric clutch hypothesis (see Lindemann, 2004). Such deformations, being asymmetric with respect to axonemal cross-sections, would not be affected by the ellipse correction approach presented here.

Relative to an unmoving external reference, an invariable CP orientation can only be achieved if the $\mathrm{CP}$ is fixed along its length. However, if the $\mathrm{CP}$ is not seen in a constant position, there are three possible explanations - one of which involves an unmoving $\mathrm{CP}$, whereas the other two require $\mathrm{CP}$ movement. First, the $\mathrm{CP}$ orientation might be invariant (i.e. unmoving) 
along the length of an individual axoneme, but variant between axonemes as a result of differences in the initial orientation of CPs at their origins. Second, the CP might be fixed at its basal end, but twist around its longitudinal axis during flagellar beating. Since the basal end is unmoving, this model requires a reverse twist of the CP during the recovery stroke to avoid super-coiling of the $\mathrm{CP}$. The net $\mathrm{CP}$ movement is thus an oscillation. Last, the CP might be movable at the base and also along its length - allowing the $\mathrm{CP}$ to rotate relative to an unmoving external reference during the beat.

Here, we report that $\mathrm{CP}$ orientation is fixed within kinetoplastid flagella and hence the $\mathrm{CP}$ does not move during flagellar beating. We used RNAi to investigate how CP orientation is defined and whether it can be freed from constraint. The fact that the $\mathrm{CP}$ is fixed in free Leishmania flagella suggests that $\mathrm{CP}$ orientation is independent of flagellar attachment to the trypanosomatid cell body. This is strengthened by our analysis of the $T$. cruzi epimastigote flagellum, which is only associated with the cell body in its proximal segments. Yet again, the fixed $\mathrm{CP}$ orientation is maintained throughout the attached and free segments of this flagellum.

The RNAi mutants described here all result in forms of flagellar paralysis. However, the orientation of the $\mathrm{CP}$ is not being entrained by flagellar beating. This can be demonstrated by the previously described RNAi against $P F R 2$, which also results in immotility in T. brucei (Bastin et al., 1998), but a careful examination of axonemal cross-sections reveals that the $\mathrm{CP}$ remains fixed. Since CP orientation is independent of flagellar attachment and also beating, it must be defined by interactions occurring solely within the flagellum. Such definition might occur by either: (1) determination of CP orientation at its origin at the basal plate, which is then passively inherited distally along the flagellum; or (2) physical constraint of CP orientation by proteins existing along the length of the axoneme. Our data suggest that the former is an absolute requirement for correct $\mathrm{CP}$ orientation; misorientation of the $\mathrm{CP}$ can be prevented only when a native basal body configuration is present in the cell, and $\mathrm{CP}$ misorientation does not seem able to be corrected even when the ultra-structure of the outer doublets appears normal (see $\gamma$ tubulin RNAi). However, there does seem to be a partial dependence on constraint by the axoneme because, in cells containing normal basal bodies but with severely disrupted axonemal configurations, correct $\mathrm{CP}$ orientation is sometimes lost. Hence, the mechanism by which the kinetoplastid CP is constrained - when it is free to move in species such as Chlamydomonas and Paramecium - acts predominantly at the birth of the $\mathrm{CP}$ at the distal-end of the basal body, but is also to a lesser degree constrained by physical contacts made by the $\mathrm{CP}$ along the length of the flagellum. Although we cannot entirely rule out movement of the $\mathrm{CP}$ in trypanosome RNAi mutants, the removal of the $\mathrm{CP}$ constraint in trypanosomes most probably does not result in CP rotation or oscillation, but in a mis-orientation that is invariant within an individual axoneme. This can be seen in the fact that all the RNAi mutants described are paralysed, and hence cannot be moving the $\mathrm{CP}$ in response to flagellar beating. Whether or not the $\mathrm{CP}$ in such axonemes would move in response to an externally enforced beat remains to be seen.

The functional reasons for $\mathrm{CP}$ rotation in some but not all axonemes have yet to be fully understood. Bray suggested that the CP rotates whenever a ciliary beat is three dimensional i.e. when the effective and recovery strokes occur in different planes (Bray, 1992). Paramecium supports this condition since it performs non-planar, asymmetrical waves - during forward or reverse swimming, the cilia beat presents a planar power stroke and a three-dimensional recovery stroke (Omoto and Kung, 1979). However, Chlamydomonas performs planar waves whether swimming forwards (asymmetrical) or backwards (symmetrical) (Bessen et al., 1980). In addition, Chlamydomonas flagella are able to propagate planar asymmetrical and symmetrical waveforms despite the lack of CP (Frey et al., 1997; Wakabayashi et al., 1997). Another proposal is that cilia and flagella of organisms that rely on rapid changes in waveform, beat frequency and effective stroke orientation require variable $\mathrm{CP}$ position, whereas an unchanging orientation is supposed to be a derived simplification in organelles that have a permanent bend plane, such as ctenophore comb plate cilia and many metazoan spermatozoa (Mitchell, 2004). However, this hypothesis also appears incomplete since trypanosomes - here shown to have a fixed $\mathrm{CP}$ orientation - present complex waveforms. The main waveform is a planar, symmetrical wave run from the distal tip to its base (Holwill, 1965a; Holwill, 1965b), but trypanosomes can also re-orientate themselves by reversing their flagellar wave (Sugrue et al., 1988), which becomes asymmetrical and runs from base to tip (Holwill and McGregor, 1976). Swimming reversal has also been reported in Chlamydomonas (Bessen et al., 1980) and Paramecium (Naitoh and Kaneko, 1973), although the mode is distinct in each case (Holwill and McGregor, 1976). The trypanosome flagellum is not only a dynamic motility organelle, but has also evolved to perform other biological processes, such as participating in cell division (Moreira-Leite et al., 2001) and moving the parasite through challenging environments during its cell cycle in the vertebrate and invertebrate host. In the invertebrate host, the flagellum is an organelle for attachment to the host epithelia (Vickerman, 1973; Vickerman and Tetley, 1990), which ensures transmission to vertebrates. Whether the trypanosome swimming behaviour is not complex enough to require movable $\mathrm{CP}$ orientation to differentially regulate specific dynein arms is not yet clear. However, it seems reasonable to suggest that the swimming changes trypanosomes have to deal with might well be precisely regulated but by means other than a variable $\mathrm{CP}$ orientation.

This report has brought further insights into the relationship of functional constraints and axoneme integrity in eukaryotic flagella. This raises interesting questions about the molecular mechanisms of such phenomena and one wonders whether differences between axonemes capable of $\mathrm{CP}$ rotation versus axonemes bearing fixed CPs could be reflected in flagellar composition. In this regard, flagellar proteome comparisons hold much promise.

\section{Materials and Methods \\ Cell lines}

Procyclic Trypanosoma brucei 427 strain cells were maintained in SDM 79 medium supplemented with $10 \% \mathrm{v} / \mathrm{v}$ foetal calf serum (FCS) at $28^{\circ} \mathrm{C}$; bloodstream-form 427 strain cells were maintained in HMI9 medium supplemented with $15 \%$ v/v FCS at $37^{\circ} \mathrm{C}$ and $5 \% \mathrm{CO}_{2}$. RNA interference (RNAi) mutant cell lines were generated from the $T$. brucei $29-13$ cell line, which is a derivative of the procyclic 427 strain containing transgenes encoding T7 RNA polymerase and tetracycline repressor 
proteins. 29-13 cells were maintained as for 427 strain procyclic cells. Stable, inducible RNAi mutants for the genes encoding PFR2, $\gamma$-tubulin and PACRG (double knockdown of A and B isoforms) have been described previously (Bastin et al., 1998; Dawe et al., 2005; McKean et al., 2003). To generate cell lines in which RNAi against the gene encoding $\delta$-tubulin could be induced, a $500 \mathrm{bp}$ fragment of the T. brucei $\delta$-tubulin open reading frame was amplified from genomic DNA using the primers 5'-CTCGAGAAGCTTCGCGAGAGTGCGGATGCTGT-3' and 5'ACTTCTCGAGAGCACTTCCCGC-3' (restriction enzyme recognition sequence underlined). This amplicon was digested with HindIII and XhoI restriction endonucleases and ligated into the vector pZJM (Wang et al., 2000). $20 \mu \mathrm{g}$ of this construct was used to stably transform $2 \times 10^{7}$ actively dividing $29-13$ cells in cold ZPFM buffer (Bellofatto and Cross, 1989) by electroporation twice at $4.25 \mathrm{kV} \mathrm{cm}^{-1}$, with three pulses of 100 microseconds separated by 200 milliseconds (Electro Square Porator; BTX). Post-electroporation, cells were allowed to recover for 16 hours, after which time stable transformants were selected by the addition of $2.5 \mu \mathrm{g} \mathrm{ml}^{-1}$ phleomycin, $15 \mu \mathrm{g} \mathrm{ml}^{-1} \mathrm{G} 418$ and $50 \mu \mathrm{g} \mathrm{ml}^{-1}$ hygromycin; and subsequently cloned by limiting dilution. Prior to induction, RNAi mutants were left in medium devoid of drug for 24 hours, then diluted to $5 \times 10^{5}$ cells ml $^{-1}$, and induced by the addition of $1 \mu \mathrm{g} \mathrm{ml}^{-1}$ tetracycline. Cultures were diluted back to a density of $5 \times 10^{5}$ cells ml ${ }^{-1}$ every 24 hours, and fresh tetracycline was added to the culture medium.

Promastigote-form Leishmania major Friedlin VI strain cells were cultured at $28^{\circ} \mathrm{C}$ in Medium 199 with Earle's Salt and L-glutamine supplemented with $40 \mathrm{mM}$ HEPES, $10 \% \mathrm{v} / \mathrm{v}$ FCS and $5 \mu \mathrm{g} \mathrm{ml}^{-1}$ haemin. Promastigote-form Herpetomonas megaseliae and choanomastigote-form Crithidia fasciculata cells were cultured in Brain and Hearth Infusion medium supplemented with 5\% v/v FCS. Epimastigoteform Trypanosoma cruzi CL Brenner strain cells were kindly supplied by J. Kelly (London School of Hygiene and Tropical Medicine, London, UK).

\section{$\delta$-tubulin for monoclonal antibody generation}

For recombinant $\delta$-tubulin expression, the T. brucei $\delta$-tubulin open reading frame was amplified from genomic DNA using the primers 5'-CATATGCCTGACAACCGTGTCGTT- ${ }^{\prime}$ and $5^{\prime}$-GGATCCTCAAGCCGCTCCGTATGCGGCAGCAGT$3^{\prime}$ (restriction enzyme recognition sequence underlined). This amplicon was ligated into the TA cloning site of pGEMT-Easy (Promega), released by NdeI and BamHI digestion, and ligated into the Escherichia coli expression vector pET15b (Novagen). Recombinant $\delta$-tubulin was expressed in FB810 (a RecA derivative of BL21) (McKean et al., 2001) and purified by Ni-affinity chromatography followed by preparative SDS-PAGE. BALB/c mice were immunised with purified recombinant $\delta$-tubulin and hybridomas produced as described previously (Woods et al., 1989).

\section{Protein sample preparation and western blotting}

For protein depletion analysis, $\delta$-tubulin RNAi mutant cells $\left(2 \times 10^{8}\right)$ were harvested at various time-points after the addition of tetracycline, washed in PBS $(150 \mathrm{mM}$ $\mathrm{NaCl}, 10 \mathrm{mM}$ sodium phosphate, $\mathrm{pH}$ 7.4) containing protease inhibitors, and resuspended in boiling Laemmli sample buffer. SDS-PAGE and immersion transfer to nitrocellulose membrane were performed using standard techniques described elsewhere (Sambrook and Russell, 2001). For immunoblotting, membranes were blocked with 3\% w/v skimmed milk in TTBS $(20 \mathrm{mM}$ Tris-HCl, $500 \mathrm{mM} \mathrm{NaCl}$, $0.05 \%$ Tween $20, \mathrm{pH} 7.5$ ), labelled with either anti- $\delta$-tubulin monoclonal antibody, or anti- $\beta$-tubulin monoclonal antibody KMX (Birkett et al., 1985), developed with horseradish peroxidase (HRP)-conjugated anti-mouse immunoglobulins (Sigma) and detected by the ECL detection system (Amersham).

\section{Sample preparation for electron microscopy}

T. brucei, T. cruzi, L. major, H. megaseliae and C. fasciculata samples for thinsection transmission electron microscopy were prepared by fixing cells in culture with $4 \%$ formaldehyde and $2 \%$ glutaraldehyde for 1 hour at room temperature. Cells were post-fixed in $1 \%$ osmium tetroxide in $100 \mathrm{mM}$ sodium phosphate buffer, $\mathrm{pH}$ 7 for 1 hour at $4^{\circ} \mathrm{C}$, washed several times in double-distilled water, stained en bloc with $1 \%$ aqueous uranyl acetate for 16 hours at $4^{\circ} \mathrm{C}$, dehydrated through acetone and embedded in epoxy resin.

\section{Geometric correction of electron micrographs}

To correct for variations in the angle of sectioning in micrographs collected in this study, the following mathematical correction was applied. Considering the axoneme as a cylinder, any cross-section perpendicular to the longitudinal axis (the $z^{\prime}$ axis) will depict a perfect circle. However, any 'glancing' section, which is not exactly $90^{\circ}$ to the $z^{\prime}$ axis, will show an ellipse whose eccentricity increases as the angle between the section and a true perpendicular section $(\theta)$ increases (see Fig. 1). Hence, an ellipse in the observed $x y$ plane - formed by taking a section at an angle $(90-\theta)^{\circ}$ to the true longitudinal axis $z^{\prime}-$ can be superimposed onto the true transverse plane, $x^{\prime} y^{\prime}$, by the transformation:

$$
\left(\begin{array}{c}
x^{\prime} \\
y^{\prime}
\end{array}\right)=\left(\begin{array}{c}
x \cos \theta \\
y
\end{array}\right),
$$

where the maximum diameter of the ellipse lies along the $x$ axis. The result is a reacquisition of a circular transverse section

From our 'transverse' sections, the positions of the centre of each A tubule of the axonemal outer doublet microtubules and the centre of each of the $\mathrm{CP}$ microtubules were mapped by hand using the software NIH Image as an interface. The position of each microtubule in the observed $x y$ plane was thus defined by a point $\left(x_{\mathrm{i}}, y_{\mathrm{i}}\right)$. The origin was defined by the mean $x_{\mathrm{i}}$ and $y_{\mathrm{i}}$ values for the nine doublets and an estimate of $\theta$ was made from the eccentricity of the doublet positions:

$$
\theta=\cos ^{-1}\left\{\frac{\min d}{\max d}\right\},
$$

where $d$ is the distance of a point from the origin, i.e.:

$$
d_{\mathrm{i}}^{2}=\left(x_{\mathrm{i}}-\bar{x}\right)^{2}+\left(y_{\mathrm{i}}-\bar{y}\right)^{2} .
$$

The axes were then rotated so that the doublet with the maximum $d_{\mathrm{i}}$ value lay along the positive $x$ axis. Microtubule positions were superimposed onto the true transverse plane $x^{\prime} y^{\prime}$ by the transformation:

$$
\left(\begin{array}{c}
x_{\mathrm{i}}^{\prime} \\
y_{\mathrm{i}}^{\prime}
\end{array}\right)=\left(\begin{array}{c}
x_{\mathrm{i}} \cos \theta \\
y_{\mathrm{i}}
\end{array}\right) .
$$

Each transformed point $\left(x^{\prime}{ }_{i}, y_{i}^{\prime}\right)$ has a distance $d^{\prime}{ }_{i}$ from the origin and makes an angle $\phi_{\mathrm{i}}$ to the $y^{\prime}$ axis. The $x^{\prime} y^{\prime}$ axes were rotated such that the mean of the differences of each $\phi_{\mathrm{i}}$ from its expected value $[2 \pi(n-1) / 9$, where $n$ is the doublet number] was zero. Finally, the microtubule positions were normalised against the mean $d^{\prime}$ value for the doublets.

For most axonemes, minimum and maximum doublet distances provide a good estimate of ellipse angle and eccentricity (see Fig. 2A). However, in situations such as the PACRG mutant cell lines, where there is gross disruption of the outer doublets, this measurement is no longer sufficient. For these axonemes, it is necessary to use information from all remaining doublets, such as fitting the data points to an ellipse. We used the free software LSM (http://www.prz.rzeszow.pl/ janand/) to perform non-linear least-squares fitting of the data. All other calculations follow as above.

The above ellipse corrections are obviously just as applicable to images as they are to individual data points (see Fig. 1C). In this case, we used parameters estimated from non-linear least-squares fitting as above and the software NIH Image (http://rsb.info.nih.gov/nih-image/) with custom macros (available from the authors of this article on request).

We thank J. Kelly for providing us with $T$. cruzi cells, F. MoreiraLeite and $\mathrm{H}$. Dawe for providing us with EM negatives, H. Lu and M. Shaw for EM assistance, and A. Baines for the assistance with monoclonal antibody production. This work has been supported by grants from the Wellcome Trust, HFSP, BBSRC and the EP Abraham Trust. K.G. is a Wellcome Trust Principal Research Fellow.

\section{References}

Bastin, P., Sherwin, T. and Gull, K. (1998). Paraflagellar rod is vital for trypanosome motility. Nature 391, 548.

Bastin, P., Pullen, T. J., Moreira-Leite, F. F. and Gull, K. (2000). Inside and outside of the trypanosome flagellum: a multifunctional organelle. Microbes Infect. 2, 1865-1874.

Bellofatto, V. and Cross, G. A. M. (1989). Expression of a bacterial gene in a trypanosomatid protozoan. Science 224, 1167-1169.

Bessen, M., Fay, R. B. and Witman, G. B. (1980). Calcium control of waveform in isolated flagellar axonemes of Chlamydomonas. J. Cell Biol. 86, 446-455.

Birkett, C. R., Foster, K. E., Johnson, L. and Gull, K. (1985). Use of monoclonal antibodies to analyse the expression of a multi-tubulin family. FEBS Lett. 187, 211218.

Bray, D. (1992). Cell Movements. New York: Garland Publishing.

Cavalier-Smith, T. (2002). The phagotrophic origin of eukaryotes and phylogenetic classification of Protozoa. Int. J. Syst. Evol. Microbiol. 52, 297-354.

Cavalier-Smith, T. and Chao, E. E.-Y. (2003). Phylogeny of Choanozoa, Apusozoa, and other Protozoa and early eukaryote megaevolution. J. Mol. Evol. 56, 540-563.

Dawe, H., Farr, H., Portman, N., Shaw, M. and Gull, K. (2005). The Parkin coregulated gene product, PACRG, is an evolutionary conserved axonemal protein that functions in outer doublet microtubule morphogenesis. J. Cell Sci. 118, 5421-5430.

Douzery, E. J. P., Snell, E. A., Bapteste, E., Delsuc, F. and Philippe, H. (2004). The timing of eukaryotic evolution: does a relaxed molecular clock reconcile proteins and fossils? Proc. Natl. Acad. Sci. USA 101, 15386-15391.

Dutcher, S. K. and Trabuco, E. C. (1998). The UNI3 gene is required for assembly of basal bodies of Chlamydomonas and encodes delta-tubulin, a new member of the tubulin superfamily. Mol. Biol. Cell 9, 1293-1308. 
Frey, E., Brokaw, C. and Omoto, C. K. (1997). Reactivation at low ATP distinguishes among classes of paralyzed flagella mutants. Cell Motil. Cytoskeleton 38, 91-99.

Fromherz, S., Giddings, T. H., Gomez-Ospina, N. and Dutcher, S. K. (2003). Mutations in $\alpha$-tubulin promote basal body maturation and flagellar assembly in the absence of $\delta$-tubulin. J. Cell Sci. 117, 303-314.

Fuge, H. (1969). Electron microscopic studies of the intraflagellar structures of trypanosomes. J. Protozool. 16, 160-166.

Garreau de Loubresse, N., Ruiz, F., Beisson, J. and Klotz, C. (2001). Role of deltatubulin and the C-tubule in assembly of Paramecium basal body. BMC Cell Biol. 2, 4.

Gibbons, B. H. and Gibbons, I. R. (1973). The effect of partial extraction of dynein arms on the movement of reactivated sea urchin sperm. J. Cell Sci. 13, 337-357.

Gibbons, I. R. and Rowe, A. J. (1965). Dynein: a protein with adenosine triphosphatase activity from cilia. Science 149, 424-426.

Goodenough, U. W. and Heuser, J. E. (1985). Substructure of inner dynein arms, radial spokes, and the central pair/projection complex of cilia and flagella. J. Cell Biol. 100, 2008-2018.

Gull, K. (1999). The cytoskeleton of trypanosomatid parasites. Annu. Rev. Microbiol. 53, 629-655.

Holwill, M. E. (1965a). Deformation of erythrocytes by trypanosomes. Exp. Cell Res. 37, 306-311.

Holwill, M. E. (1965b). The motion of Strigomonas oncopelti. J. Exp. Biol. 42, 125-137.

Holwill, M. E. and McGregor, J. L. (1976). Effects of calcium on flagellar movement in the trypanosome Crithidia oncopelti. J. Exp. Biol. 65, 229-242.

Hosokawa, Y. and Miki-Noumura, T. (1987). Bending motion of Chlamydomonas axonemes after extrusion of central-pair microtubules. J. Cell Biol. 105, 1297-1301.

Lindemann, C. B. (2004). Testing the geometric clutch hypothesis. Biol. Cell 96, 681690.

Maga, J. A., Sherwin, T., Francis, S., Gull, K. and LeBowitz, J. H. (1999). Genetic dissection of the Leishmania paraflagellar rod, a unique flagellar cytoskeleton structure. J. Cell Sci. 112, 2753-2763.

McKean, P. G., Keen, J. K., Smith, D. F. and Benson, F. E. (2001). Identification and characterisation of a RAD51 gene from Leishmania major. Mol. Biochem. Parasitol. 115, 209-216.

McKean, P. G., Baines, A., Vaughan, S. and Gull, K. (2003). $\gamma$-tubulin functions in the nucleation of a discrete subset of microtubules in the eukaryotic flagellum. Curr. Biol. 13, 598-602.

Mitchell, D. R. (2003). Orientation of the central pair complex during flagellar bend formation in Chlamydomonas. Cell Motil. Cytoskeleton 56, 120-129.

Mitchell, D. R. (2004). Speculations on the evolution of 9+2 organelles and the role of the central pair microtubules. Biol. Cell 96, 691-696.

Mitchell, D. R. and Nakatsugawa, M. (2004). Bend propagation drives central pair rotation in Chlamydomonas reinhardtii flagella. J. Cell Biol. 166, 709-715.

Moreira-Leite, F. F., Sherwin, T., Kohl, L. and Gull, K. (2001). A trypanosome structure involved in transmitting cytoplasmic information during cell division. Science 294, 587-591.

Naitoh, Y. and Kaneko, H. (1973). Control of ciliary activities by adenosine triphosphate and divalent cations in triton-extracted models of Paramecium caudatus. J. Exp. Biol. 58, 657-676.

Nigg, E. A. (2004). Centrosomes in Development and Disease. Viernheim: Wiley-VCH Verlag.

Omoto, C. K. and Kung, C. (1979). The pair of central tubules rotates during ciliary beat in Paramecium. Nature 279, 532-534.

Omoto, C. K. and Kung, C. (1980). Rotation and twist of the central-pair microtubules in the cilia of Paramecium. J. Cell Biol. 87, 33-46.

Porter, M. E. and Sale, W. S. (2000). The $9+2$ axoneme anchors multiple inner arm dyneins and a network of kinases and phosphatases that control motility. J. Cell Biol. 151, F37-F42.

Sale, W. S. (1986). The axonemal axis and calcium-induced asymmetry of active microtubule sliding of sea urchin sperm tails. J. Cell Biol. 102, 2042-2052.

Sale, W. S. and Satir, P. (1977). Direction of active sliding of microtubules in Tetrahymena cilia. Proc. Natl. Acad. Sci. USA 74, 2045-2049.

Sambrook, J. and Russell, D. W. (2001). Molecular Cloning: A Laboratory Manual. NY: Cold Spring Harbor Laboratory Press.

Silflow, C. D., Liu, B., LaVoie, M., Richardson, E. A. and Palevitz, B. A. (1999). $\gamma$ tubulin in Chlamydomonas: characterization of the gene and localization of the gene product in cells. Cell Motil. Cytoskeleton 42, 285-297.

Smith, E. F. and Lefebvre, P. A. (1997). The role of central apparatus components in the flagellar motility and microtubule assembly. Cell Motil. Cytoskeleton 38, 1-8.

Smith, E. F. and Yang, P. (2004). The radial spokes and central apparatus. Cell Motil. Cytoskeleton 57, 8-17.

Sugrue, P., Hirons, M. R., Adam, J. U. and Holwill, M. E. (1988). Flagellar wave reversal in the kinetoplastid flagellate Crithidia oncopelti. Biol. Cell 63, 127-131.

Tamm, S. L. and Tamm, S. (1981). Ciliary reversal without rotation of axonemal structures in ctenophore comb plates. J. Cell Biol. 89, 495-509.

Vickerman, K. (1973). The mode of attachment of Trypanosoma vivax in the proboscis of the tsetse fly Glossina fuscipes: an ultrastructural study of the epimastigote stage of the trypanosome. J. Protozool. 20, 394-404.

Vickerman, K. and Tetley, L. (1990). Flagellar surfaces of parasitic protozoa and their role in attachment. In Ciliary and Flagellar Membranes (ed. R. A. Bloodgood), pp. 267-304. New York: Plenum Press.

Wakabayashi, K., Yagi, T. and Kamiya, R. (1997). $\mathrm{Ca}^{2+}$-dependent waveform conversion in the flagellar axoneme of Chlamydomonas mutants lacking the centralpair/radial spokes system. Cell Motil. Cytoskeleton 38, 22-28.

Wang, Z., Morris, J. C., Drew, M. E. and Englund, P. T. (2000). Inhibition of Trypanosoma brucei gene expression by RNA interference using an integratable vector with opposing T7 promoters. J. Biol. Chem. 275, 40174-40179.

Warner, F. D. and Satir, P. (1974). The structural basis of ciliary bend formation. J. Cell Biol. 63, 35-63.

Wiese, M., Kuhn, D. and Grünfelder, C. G. (2003). Protein kinase involved in flagellarlength control. Eukaryotic Cell 2, 769-777.

Woods, A., Sherwin, T., Sasse, R., MacRae, T. H., Maines, A. J. and Gull, K. (1989). Definition of individual components within the cytoskeleton of Trypanosoma brucei by a library of monoclonal antibodies. J. Cell Sci. 93, 491-500. 